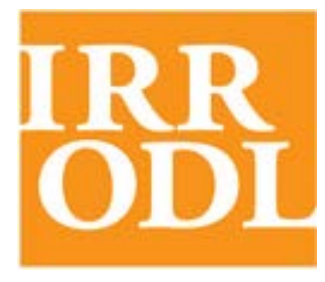

International

Review of

Research in Open

and Distance

Learning

Vol. 12.1

January - 2011

\title{
Editorial
}

\section{The Landscape of Prior Learning Assessment: A Sampling from a Diverse Field}

\author{
Dianne Conrad \\ IRRODL Special Issue Editor
}

We live today, as educators and global citizens, in a time of convergence, due in great part to the phenomenal rise of social media and networking tools that have reduced barriers to and boundaries between exchange and domains, beyond what our early trail-blazing distance educators could even have imagined. This special issue of IRRDOL features another exciting convergence - one that represents the somewhat narrow overlap of interests between open and distance learning (ODL) and the recognition of prior learning (RPL). In this special issue, therefore, we offer a selection of articles and practitioner-based reports that, in varying measures, span both fields.

Two important foundational issues underlying the prior learning field should be addressed here. The first concerns language. Prior learning recognition, although practiced globally, has a wide variety of labels and acronyms. In Canada, we are most likely to refer to it as PLAR - prior learning assessment and recognition. RPL - recognition of prior learning - is also beginning to become popular in certain Canadian jurisdictions. In the United States, PLA - prior learning assessment - is the most common label. Around the rest of the world, the shared understanding that informally or experientially attained learning can be recognized for credit toward credentialization is referred to as the assessment of prior learning (APL), the assessment/accreditation of prior and experiential learning (APEL), and a variety of other similar terms. You will notice, in the articles in this issue, a variety in terminology that would logically accompany an international publication. This short editorial uses the PLAR acronym. 
A second important issue concerns the place of PLAR in the world of open and distance learning. Given that the mandate of both prior learning assessment and open universities is to reduce barriers to learning and provide learning possibilities to a wider audience, it makes sense that the two initiatives should coalesce in practice. While this is sometimes the case, it is not always the case - and the inclusion of robust prior learning and assessment practices at university level even in distance institutions is far less common than PLAR champions would like.

IRRODL's dedication, therefore, of a special issue devoted to PLAR issues within the ODL context is both exciting and challenging. And as a practitioner and researcher in the field, I have been privileged and enlightened to have had this opportunity to engage with the work of my colleagues in Canada, in the United States, and in Europe.

PLAR is, in itself, not a discipline; and whether or not it even constitutes a field of study is questionable. Certainly it is a child of adult education, springing historically from the works of Dewey, Kolb, and Knowles; but as a practice, PLAR's parameters are porous, and its principles extend out and enmesh into adult education's other sub-areas, including workplace learning, informal learning, assessment, vocational education, and, more recently and perhaps urgently, foreign credential recognition and training. Issues of policy in all these areas, together with economic and social concerns, are also related and integral to PLAR research, practice, and literature.

The broad span of PLAR interests is evident in this issue's collection of articles and field notes. Interestingly, the field notes - IRRODL's section of reports on work-in-progress research or onthe-ground work - provide a more centralized and consistent look at the field than do the five research articles. From the field notes, a rich landscape of PLAR initiatives emerges. Work from both Canada and the United States is highlighted, with Brigham and Klein-Collins' piece describing the PLAR mother-organization CAEL's (the US Council for Adult and Experiential Learning) latest broad initiative and Wihak's Canadian piece describing a recent pan-Canadian scholarly initiative. The remaining field notes describe a number of projects at various PLARfriendly institutions, ranging from Travers and Evans' proposed PLA evaluation framework at Empire State College in New York to Santa Mina and her colleagues' description of their work with internationally educated nurses at Ryerson University in Ontario. Gordon, Ireland, and Wong also describe a PLAR IEN project.

The longer articles comprise a varied collection of PLAR-related interests. From Europe, Olaf Zawacki-Richter and his colleagues Eva Maria Bäcker and Anke Hanft present the results of a research study which explores how the competences demonstrated in e-portfolio preparation can be extrapolated for use in blended learning. Zawacki et al.'s research reflects Europe's ongoing focus on e-portfolio use in areas related to prior learning, competencies, and credential recognition.

From the United States, Barry University's Judith Brown also considers the place of e-portfolios in PLAR work as she examines their potential in promoting connections between several different types of learning - academic, workplace, and web-based. Brown contends that this type of PLAR 
enables undergraduate adult learners to not only articulate and equate experiential learning to academic knowledge but also to demonstrate knowledge visually and audibly through the electronic medium.

Linda Salter's research describes the first phase of her two-phase, mixed-method doctoral study. The study reaches into foundational adult education aspects of prior learning, exploring how and to what extent adults' willingness to engage in learning in mature adulthood is influenced by prior experiences and specific individual personality variables, such as perceived locus of control and degree of self-efficacy. Salter's study provides useful insight into some of adult learning's many realms; in this case, the connection between prior learning and adult learning efficiencies is explored. Salter's notion of personality variables as factors in learning performance echoes, somewhat, Zawacki-Richter et al.'s exploration of learning types that manifest in blended learning formats. Both leave the door open for further research in these intriguing areas.

The next two groups of contributors, each from an institution that makes extensive use of prior learning practices, offer detailed insights into practice. Sara Leiste and Kathryn Jensen present an overview of Capella University's broad PLA practice, calling specific attention to their use of the online PLA lab to assist learners with portfolio preparation. As with most ardent PLA practices, Capella's system is built on CAEL's widely adopted 10 standards of good practice.

Empire State College's PLA process also adheres to CAEL standards. Describing their very robust practice, which consistently involves thousands of faculty advisors, evaluators, and learners, Nan Travers and her colleagues focus more specifically on one aspect of prior learning assessment - the language of evaluation. Their research outlines various ways in which college evaluators describe the learning presented to them in learners' portfolios. This fascinating area of study points to the many factors that underpin the nature of assessment. Although regarded by many as an unassailable entity, Travers et al.'s research demonstrates the porousness of the prior learning evaluation process and, by extension, of all assessment practices.

From Canada, a unique perspective on PLAR practice at the college level is presented by Lloyd Hawkeye Robertson, who draws on his experience as a counselling practitioner in Saskatchewan's north to examine the appropriate role of PLAR facilitators in guiding and/or encouraging participants' reflections. While Robertson brings forward issues around concepts of spirituality and Aboriginal engagement with PLAR practice in a specific context, his thoughtful piece raises ethical questions that have been heard before in discussions of practice in other countries, notably South Africa.

Also from Canada, but drawing on data from an international study, Dianne Conrad investigates the role of language in portfolio learning. As with all learning and interaction at a postsecondary level, the PLAR experience brings learners face-to-face with new uses of language and the need to adapt to new ways of thinking and writing about their experiences. Conrad's research resonates with Nan Travers and colleagues' recent work on the language of assessment, which is also published in this special PLAR edition of IRRODL. 
Taken together, the tally of research and field-note topics comprises an eclectic mix. What is indisputable, however, is the consistent return to issues of learning and pedagogy that permeate PLAR discussions. As PLAR grows in stature and continues to lobby for support and acceptance within the postsecondary environment, its contribution and connection to sound learning practice is critical. Its pedagogical role must not only be seen but also shared, discussed, and debated. Its literature must be developed and critically explored. I hope that this issue of IRRODL has contributed to that process.

Editorial revised October 2011

\section{Athabasca University $\mathbf{I}$}

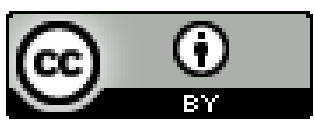

